\title{
Sandwich synchronization of memristor-based hyperchaos systems with time delays
}

\author{
Hongjuan $\mathrm{Wu}^{1 *}$, Jiang Xiong ${ }^{1}$, Xiang $\mathrm{Hu}^{1}$, Yuming Feng ${ }^{1}$ and Liangliang $\mathrm{Li}^{2}$
}

\author{
"Correspondence: \\ juan10329@163.com \\ 'School of Computer Science and \\ Engineering, Chongqing Three \\ Gorges University, Wanzhou, \\ Chongqing 404120, P.R. China \\ Full list of author information is \\ available at the end of the article
}

\begin{abstract}
In this paper, a memristor-based hyperchaotic system is introduced. Considering time delays between the drive system and the response system in the process of synchronization, this paper designs one kind of flexible sandwich controller, which includes a rest in the sandwich structure, to realize the synchronization between two memristor-based hyperchaotic systems. Based on Lyapunov stability theory, matrix inequality, sandwich control and considering time delays, the exponential synchronization conditions for the memristor-based hyperchaotic systems with time delays via sandwich control are given. Finally, simulation results are displayed to verify the effectiveness and feasibility of this method.
\end{abstract}

Keywords: memristor-based; hyperchaotic system; time delays; synchronization; sandwich control

\section{Introduction}

Memristor as the fourth fundamental circuit element was first proposed by Chua [1] in 1971 based on logical symmetry arguments, and it was realized by Hewlett-Packard [2] research team in 2008. This passive electronic device has generated unprecedented worldwide interest because of its potential applications in signal processing, programmable logic, control system, neural network, brain-computer interface [3], etc.

Recently, the research on memristor-based circuits is becoming a hot topic. A lot of memristor oscillator systems have been used in generating signals which are found in satellite communications, radio, switching power supply, etc. [4-10]. With the potential memristor applications, it is necessary to do some deep research on the related nonlinear memristor-based oscillator systems [11-13]. Itoh and Chua [14] derived several nonlinear oscillators from Chua's oscillators by replacing Chua's diodes with memristors. Bao et al. $[15,16]$ studied the complicated dynamical behaviors of the memristor oscillators. Although various memristor-based chaotic systems have been researched in recent years [17-19], the research of synchronization between two memristor-based hyperchaotic systems is rarely reported. Because the synchronization of the memristor-based chaotic systems is a challenging problem [20-23], chaotic behavior, especially the hyperchaotic behavior that has more than one positive Lyapunov exponent, may be uncoordinated and unpredictable.

(c) The Author(s) 2018. This article is distributed under the terms of the Creative Commons Attribution 4.0 International License (http://creativecommons.org/licenses/by/4.0/), which permits unrestricted use, distribution, and reproduction in any medium, provided you give appropriate credit to the original author(s) and the source, provide a link to the Creative Commons license, and indicate if changes were made. 
Sandwich control is one kind of discontinuous control. It can be used in many industrial fields [24]. It could include many subsystems that are continuous. Feng et al. [25] studied the sandwich structure control system that includes two continuous controls and an impulsive control in each period and applied it to control Chua's oscillator. While this paper will talk about another kind of flexible sandwich structure, which is different from [25]. In each period of this sandwich control system, the first and third parts of the control system are continuous controls, which may be continuous controls with different control gains. Between these two parts, there is a rest. This kind of sandwich control structure is very suitable for these systems that cannot be controlled continuously all the time.

In this paper, we apply this kind of sandwich control to ensure the synchronization between two memristor-based hyperchaotic systems. We pay attention to time delays between the drive system and the response system when we control the error system [2629], because there are always some transmission time delays between the drive system and the response system in the real environment. Based on Lyapunov stability theory, matrix inequality, sandwich control and considering time delays, the exponential synchronization conditions for the memristor-based hyperchaotic systems with time delays via sandwich control are given.

\section{The fourth-order memristor-based hyperchaotic system}

Memristor is a nonlinear circuit element, and its value is not unique. Assume that the fluxcontrolled memristor is characterized by the mathematical model of a smooth continuous cubic monotone-increasing nonlinearity [15]

$$
q(\varphi)=a \varphi+b \varphi^{3}
$$

where $a$ and $b$ are parameters. From equation (1), the memductance $W(\varphi)$ is obtained as follows:

$$
W(\varphi)=d q(\varphi) / d \varphi=a+3 b \varphi^{2} .
$$

Consider one kind of fourth-order memristor-based hyperchaotic oscillator system as Figure 1 shows. It is directly extended from Chua's oscillator by replacing Chua's diode with a smooth flux-controlled memristor and a negative conductance [14, 30, 31]. In this memristor-based hyperchaotic circuit, these two parts, passive memristor (fluxcontrolled memristor) and negative conductance, can be considered an active memristor.

According to KCL and KVL, this circuit can be described by the following differential equations.

$$
\left\{\begin{array}{l}
\dot{V}_{1}(t)=\frac{1}{C_{1} R_{1}}\left(V_{2}(t)-V_{1}(t)\right)+\frac{G}{C_{1}} V_{1}(t)-\frac{1}{C_{1}} W(\varphi(t)) V_{1}(t), \\
\dot{V}_{2}(t)=\frac{1}{C_{2} R_{1}} V_{1}(t)-\frac{1}{C_{2} R_{1}} V_{2}(t)+\frac{1}{C_{2}} i(t), \\
\dot{i}(t)=-\frac{1}{L} V_{2}(t)-\frac{R_{2}}{L} i(t), \\
\dot{\varphi}(t)=V_{1}(t),
\end{array}\right.
$$

where $C$ refers to capacitor, $V$ denotes voltages, $W(\varphi)$ is memductance, $R$ denotes resistors, $\varphi, L, i, G$ are magnetic flux, inductor, current and conductance, respectively. From 




Figure 1 Memristor-based hyperchaotic system.

equations (2) and (3), it follows that

$$
\left\{\begin{array}{l}
\dot{V}_{1}(t)=\frac{1}{C_{1} R_{1}} V_{2}(t)-\left(\frac{1}{C_{1} R_{1}}-\frac{G}{C_{1}}+\frac{a}{C_{1}}\right) V_{1}(t)-\frac{3 b}{C_{1}} \varphi(t)^{2} V_{1}(t) \\
\dot{V}_{2}(t)=\frac{1}{C_{2} R_{1}} V_{1}(t)-\frac{1}{C_{2} R_{1}} V_{2}(t)+\frac{1}{C_{2}} i(t) \\
\dot{i}(t)=-\frac{1}{L} V_{2}(t)-\frac{R_{2}}{L} i(t) \\
\dot{\varphi}(t)=V_{1}(t)
\end{array}\right.
$$

If we let $x_{1}=V_{1}, x_{2}=V_{2}, x_{3}=i, x_{4}=\varphi, \gamma_{1}=\frac{1}{C_{1} R_{1}}, \gamma_{2}=\frac{1}{C_{1} R_{1}}-\frac{G}{C_{1}}+\frac{a}{C_{1}}, \gamma_{3}=\frac{3 b}{C_{1}}, \gamma_{4}=\frac{1}{C_{2} R_{1}}$, $\gamma_{5}=\frac{1}{C_{2}}, \gamma_{6}=\frac{1}{L}$ and $\gamma_{7}=\frac{R_{2}}{L}$, system (4) can be further expressed as follows:

$$
\left\{\begin{array}{l}
\dot{x}_{1}(t)=\gamma_{1} x_{2}(t)-\gamma_{2} x_{1}(t)-\gamma_{3} x_{4}(t)^{2} x_{1}(t) \\
\dot{x}_{2}(t)=\gamma_{4} x_{1}(t)-\gamma_{4} x_{2}(t)+\gamma_{5} x_{3}(t) \\
\dot{x}_{3}(t)=-\gamma_{6} x_{2}(t)-\gamma_{7} x_{3}(t) \\
\dot{x}_{4}(t)=x_{1}(t)
\end{array}\right.
$$

If we set $\gamma_{1}=15, \gamma_{2}=-3.2, \gamma_{3}=19.7, \gamma_{4}=1, \gamma_{5}=1, \gamma_{6}=15, \gamma_{7}=0.52$ for the initial states $\left(10^{-4}, 10^{-4}, 10^{-4}, 10^{-4}\right)^{T}$, by means of a computer program with MATLAB 7.0, computer simulation shows that system (5) has hyperchaotic attractors as shown in Figure 2.

Remark 1 Although various memristor-based chaotic systems have been researched extensively in recent years, the research of memristor-based hyperchaotic systems is rarely reported and investigated directly. Thus the hyperchaotic system (5) is important for understanding of memristor-based hyperchaotic systems. 

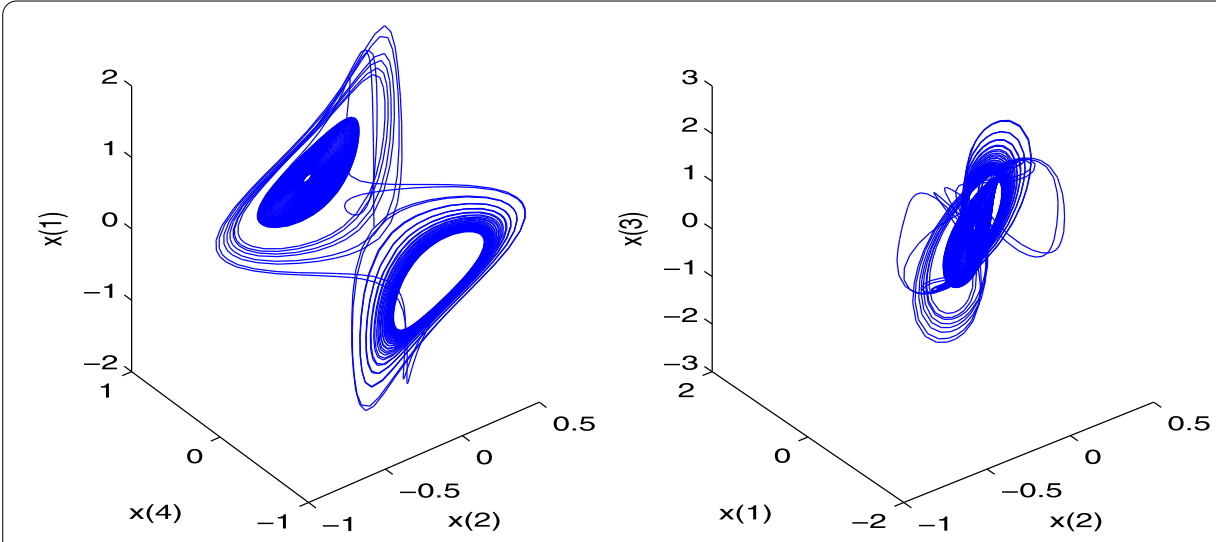

Figure 2 Memristor-based hyperchaotic attractors: 3D projection.

\section{Synchronization of the memristor-based hyperchaotic systems with time delays}

In this section, system (5) is taken as two parts, that is,

$$
\dot{x}(t)=A x(t)+B g(x(t)),
$$

where $x(t)=\left(x_{1}(t), x_{2}(t), x_{3}(t), x_{4}(t)\right)^{T}$,

$$
A=\left[\begin{array}{cccc}
-\gamma_{2} & \gamma_{1} & 0 & 0 \\
\gamma_{4} & -\gamma_{4} & \gamma_{5} & 0 \\
0 & -\gamma_{6} & -\gamma_{7} & 0 \\
1 & 0 & 0 & 0
\end{array}\right], \quad B=\left[\begin{array}{cccc}
-\gamma_{3} & 0 & 0 & 0 \\
0 & 0 & 0 & 0 \\
0 & 0 & 0 & 0 \\
0 & 0 & 0 & 0
\end{array}\right] \quad \text { and } g(x(t))=\left[\begin{array}{c}
x_{4}^{2} x_{1} \\
0 \\
0 \\
0
\end{array}\right]
$$

Because $g(x)$ satisfies the Lipschitz condition, for any $x, x^{\prime} \in \Omega$, we have

$$
\left|g_{i}(x)-g_{i}\left(x^{\prime}\right)\right| \leq L\left|x-x^{\prime}\right|, \quad i=1,2,3,4,
$$

where $L$ is the Lipschitz coefficient.

If we take system (6) as the drive system, the response system is described by

$$
\dot{y}(t)=A y(t)+B g(y(t))+u(t)
$$

where $y$ is the state variable, $y(t)=\left(y_{1}(t), y_{2}(t), y_{3}(t), y_{4}(t)\right)^{T} \cdot u(t)$ is the sandwich controller, which might be with two different control gains. Considering time delays between the drive system and the response system, $u(t)$ can be described as the following equations:

$$
u(t)= \begin{cases}I_{1}(y(t)-x(t-\tau)), & n T \leq t<n T+\theta_{1} T, \\ 0, & n T+\theta_{1} T \leq t<n T+\left(\theta_{1}+\theta_{2}\right) T, \\ I_{2}(y(t)-x(t-\tau)), & n T+\left(\theta_{1}+\theta_{2}\right) T \leq t<(n+1) T,\end{cases}
$$

where $I_{1}$ and $I_{2}$ refer to control gains, $\theta_{1}$ and $\theta_{2}$ are the percentages of each period $T$, $\theta_{1}+\theta_{2}<1$. Let $e(t)=y(t)-x(t-\tau)$, then $e(t)$ is the synchronization error between system 
(6) and system (8) with time delays. The error system can be described by the following equation:

$$
\dot{e}(t)=\dot{y}(t)-\dot{x}(t-\tau)=A e(t)+B(g(y(t))-g(x(t-\tau)))+u(t) .
$$

If we apply sandwich control to system (10), then the error system can be re-described as three subsystems:

$$
\begin{cases}\dot{e}(t)=A e(t)+B(g(y(t))-g(x(t-\tau)))+I_{1} e(t), & n T \leq t<n T+\theta_{1} T \\ \dot{e}(t)=A e(t)+B(g(y(t))-g(x(t-\tau))), & n T+\theta_{1} T \leq t<n T+\left(\theta_{1}+\theta_{2}\right) T, \\ \dot{e}(t)=A e(t)+B(g(y(t))-g(x(t-\tau)))+I_{2} e(t), & n T+\left(\theta_{1}+\theta_{2}\right) T \leq t<(n+1) T .\end{cases}
$$

Remark 2 In the real environment, there are always some time delays between the drive system and the response system. Thus considering time delays between the drive system and the response system in the process of synchronization is of great practical significance.

Remark 3 The sandwich control put forward by this paper is a general model, which can be used as a prototype of other discontinuous controls that include more than two continuous controls with different control gains in each period.

Lemma 1 ([32]) Given any real matrices $\Sigma_{1}, \Sigma_{2}, \Sigma_{3}$ of appropriate dimensions and a scalar $\varepsilon>0$ such that $0<\Sigma_{3}=\Sigma_{3}^{T}$, the following inequality holds:

$$
\Sigma_{1}^{T} \Sigma_{2}+\Sigma_{2}^{T} \Sigma_{1} \leq \varepsilon \Sigma_{1}^{T} \Sigma_{3} \Sigma_{1}+\varepsilon^{-1} \Sigma_{2}^{T} \Sigma_{3}^{-1} \Sigma_{2}
$$

Next, we will find the proper $T, I_{1}, I_{2}, \theta_{1}, \theta_{2}, s_{1}, s_{2}, s_{3}$ to ensure the synchronization between drive system (6) and response system (8). In other words, if the stability of error system (11) can be guaranteed, drive system (6) and response system (8) can realize synchronization.

Theorem 1 Suppose there are three positive scalars $\left(s_{1}>0, s_{2}>0, s_{3}>0, \varepsilon_{1}>0, \varepsilon_{2}>0\right.$ and $\left.\varepsilon_{3}>0\right)$ and the following conditions hold:

(1) $A+A^{T}+2 I_{1} E+\varepsilon_{1} B B^{T}+\varepsilon_{1}^{-1} \tilde{L}^{2} E+s_{1} E \leq 0$,

(2) $A+A^{T}+\varepsilon_{2} B B^{T}+\varepsilon_{2}^{-1} \tilde{L}^{2} E-s_{2} E \leq 0$,

(3) $A+A^{T}+2 I_{2} E+\varepsilon_{3} B B^{T}+\varepsilon_{3}^{-1} \tilde{L}^{2} E+s_{3} E \leq 0$,

(4) $s_{1} \theta_{1}-s_{2} \theta_{2}+s_{3}\left(1-\theta_{1}-\theta_{2}\right)>0$, where $\tilde{L}$ is the largest Lipschitz coefficient, then error system (11) is exponentially stable. That is, the exponential synchronization between system (6) and system (8) with time delays will be realized.

Proof Define a Lyapunov function $V(e(t))=e(t)^{T} e(t)$. When $n T \leq t<n T+\theta_{1} T$, the derivative of $V(e(t))$ with respect to time $t$ of the first subsystem is calculated and estimated as follows:

$$
\begin{aligned}
\dot{V}(e(t)) & =2 e(t)^{T} \dot{e}(t)=2 e(t)^{T}\left(A e(t)+B(g(y(t))-g(x(t-\tau)))+I_{1} e(t)\right) \\
& =2 e(t)^{T} A e(t)+2 e(t)^{T} B(g(y(t))-g(x(t-\tau)))+2 e(t)^{T} I_{1} e(t) \\
& =e(t)^{T}\left(A+A^{T}\right) e(t)+2 e(t)^{T} I_{1} e(t)+2 e(t)^{T} B(g(y(t))-g(x(t-\tau))) .
\end{aligned}
$$


Through Lemma 1, we get

$$
\begin{aligned}
& 2 e(t)^{T} B(g(y(t))-g(x(t-\tau))) \\
& \quad \leq \varepsilon_{1}(B e(t))^{T} B e(t)+\varepsilon_{1}^{-1}(g(y(t))-g(x(t-\tau)))^{T}(g(y(t))-g(x(t-\tau))) \\
& \quad=\varepsilon_{1} e(t)^{T} B B^{T} e(t)+\varepsilon_{1}^{-1}\|g(y(t))-g(x(t-\tau))\|^{2} \\
& \quad \leq \varepsilon_{1} e(t)^{T} B B^{T} e(t)+\varepsilon_{1}^{-1} \tilde{L}^{2} e(t)^{T} e(t) .
\end{aligned}
$$

So that the value of $\dot{V}(e(t))$ should satisfy

$$
\begin{aligned}
\dot{V}(e(t)) & \leq e(t)^{T}\left(A+A^{T}+2 I_{1} E+\varepsilon_{1} B B^{T}\right) e(t)+\varepsilon_{1}^{-1} \tilde{L}^{2} e(t)^{T} e(t) \\
& =e(t)^{T}\left(A+A^{T}+2 I_{1} E+\varepsilon_{1} B B^{T}+\varepsilon_{1}^{-1} \tilde{L}^{2} E+s_{1} E\right) e(t)-s_{1} V(e(t)) \\
& \leq-s_{1} V(e(t)) .
\end{aligned}
$$

Similarly, when $n T+\theta_{1} T \leq t<n T+\left(\theta_{1}+\theta_{2}\right) T$, the derivative of $V(e(t))$ with respect to time $t$ of the second subsystem is as follows:

$$
\begin{aligned}
\dot{V}(e(t)) & =2 e(t)^{T} \dot{e}(t)=e(t)^{T}\left(A+A^{T}\right) e(t)+2 e(t)^{T} B(g(y(t))-g(x(t-\tau))) \\
& \leq e(t)^{T}\left(A+A^{T}\right) e(t)+\varepsilon_{2} e(t)^{T} B B^{T} e(t)+\varepsilon_{2}^{-1}\|g(y(t))-g(x(t-\tau))\|^{2} \\
& \leq e(t)^{T}\left(A+A^{T}+\varepsilon_{2} B B^{T}\right) e(t)+\varepsilon_{2}^{-1} \tilde{L}^{2} e(t)^{T} e(t) \\
& =e(t)^{T}\left(A+A^{T}+\varepsilon_{2} B B^{T}+\varepsilon_{2}^{-1} \tilde{L}^{2} E-s_{2} E\right) e(t)+s_{2} V(e(t)) \\
& \leq s_{2} V(e(t)) .
\end{aligned}
$$

When $n T+\left(\theta_{1}+\theta_{2}\right) T \leq t<(n+1) T$, the $\dot{V}(e(t))$ of the third subsystem is as follows:

$$
\begin{aligned}
\dot{V}(e(t)) & =2 e(t)^{T} \dot{e}(t) \leq e(t)^{T}\left(A+A^{T}+2 I_{2} E+\varepsilon_{3} B B^{T}\right) e(t)+\varepsilon_{3}^{-1} \tilde{L}^{2} e(t)^{T} e(t) \\
& =e(t)^{T}\left(A+A^{T}+2 I_{2} E+\varepsilon_{3} B B^{T}+\varepsilon_{3}^{-1} \tilde{L}^{2} E+s_{3} E\right) e(t)-s_{3} V(e(t)) \\
& \leq-s_{3} V(e(t)) .
\end{aligned}
$$

Therefore, we get that

Case 1. When $n=0$, then

Subcase 1 . If $0 \leq t<\theta_{1} T$, then we have that

$$
\begin{aligned}
& V(e(t)) \leq V\left(e\left(t_{0}\right)\right) \exp \left(-s_{1} t\right) \\
& V\left(e\left(\theta_{1} T^{-}\right)\right) \leq V\left(e\left(t_{0}\right)\right) \exp \left(-s_{1} \theta_{1} T\right)
\end{aligned}
$$

Subcase 2. If $\theta_{1} T \leq t<\left(\theta_{1}+\theta_{2}\right) T$, then we have that

$$
\begin{aligned}
& \begin{aligned}
V(e(t)) & \leq V\left(e\left(\theta_{1} T^{-}\right)\right) \exp \left(s_{2}\left(t-\theta_{1} T\right)\right) \\
& \leq V\left(e\left(t_{0}\right)\right) \exp \left(-s_{1} \theta_{1} T+s_{2}\left(t-\theta_{1} T\right)\right),
\end{aligned} \\
& V\left(\left(\theta_{1}+\theta_{2}\right) T^{-}\right) \leq V\left(e\left(t_{0}\right)\right) \exp \left(-s_{1} \theta_{1} T+s_{2} \theta_{2} T\right) .
\end{aligned}
$$


Subcase 3. If $\left(\theta_{1}+\theta_{2}\right) T \leq t<T$, then we have that

$$
\begin{gathered}
V(e(t)) \leq V\left(e\left(\left(\theta_{1}+\theta_{2}\right) T^{-}\right)\right) \exp \left(-s_{3}\left(t-\theta_{1} T-\theta_{2} T\right)\right) \\
\leq V\left(e\left(t_{0}\right)\right) \exp \left(-s_{1} \theta_{1} T+s_{2} \theta_{2} T-s_{3}\left(t-\theta_{1} T-\theta_{2} T\right)\right), \\
V\left(e\left(T^{-}\right)\right) \leq V\left(e\left(t_{0}\right)\right) \exp \left(-s_{1} \theta_{1} T+s_{2} \theta_{2} T-s_{3}\left(T-\theta_{1} T-\theta_{2} T\right)\right) .
\end{gathered}
$$

Similarly, we get that

Case 2. When $n=1$, then

Subcase 1 . If $T \leq t<T+\theta_{1} T$, then we have that

$$
\begin{aligned}
& V(e(t)) \leq V\left(e\left(t_{0}\right)\right) \exp \left(-s_{1} \theta_{1} T+s_{2} \theta_{2} T-s_{3}\left(T-\theta_{1} T-\theta_{2} T\right)-s_{1}(t-T)\right) \\
& V\left(e\left(\left(T+\theta_{1} T\right)^{-}\right)\right) \leq V\left(e\left(t_{0}\right)\right) \exp \left(-2 s_{1} \theta_{1} T+s_{2} \theta_{2} T-s_{3}\left(T-\theta_{1} T-\theta_{2} T\right)\right)
\end{aligned}
$$

Subcase 2 . If $T+\theta_{1} T \leq t<T+\left(\theta_{1}+\theta_{2}\right) T$, then we have that

$$
\begin{aligned}
& V(e(t)) \leq V\left(e\left(t_{0}\right)\right) \exp \left(-2 s_{1} \theta_{1} T+s_{2} \theta_{2} T-s_{3}\left(T-\theta_{1} T-\theta_{2} T\right)+s_{2}\left(t-T-\theta_{1} T\right)\right) \\
& V\left(e\left(\left(T+\left(\theta_{1}+\theta_{2}\right) T\right)^{-}\right)\right) \leq V\left(e\left(t_{0}\right)\right) \exp \left(-2 s_{1} \theta_{1} T+2 s_{2} \theta_{2} T-s_{3}\left(T-\theta_{1} T-\theta_{2} T\right)\right)
\end{aligned}
$$

Subcase 3. If $T+\left(\theta_{1}+\theta_{2}\right) T \leq t<2 T$, then we have that

$$
\begin{aligned}
V(e(t)) \leq & V\left(e\left(t_{0}\right)\right) \\
& \times \exp \left(-2 s_{1} \theta_{1} T+2 s_{2} \theta_{2} T-s_{3}\left(T-\theta_{1} T-\theta_{2} T\right)-s_{3}\left(t-T-\theta_{1} T-\theta_{2} T\right)\right), \\
V\left(e\left(2 T^{-}\right)\right) & \leq V\left(e\left(t_{0}\right)\right) \exp \left(-2 s_{1} \theta_{1} T+2 s_{2} \theta_{2} T-2 s_{3}\left(T-\theta_{1} T-\theta_{2} T\right)\right) .
\end{aligned}
$$

By induction, we get the following.

Case $m+1$. When $n=m$, then

Subcase 1. If $m T \leq t<m T+\theta_{1} T$, then we have that

$$
V(e(t)) \leq V\left(e\left(t_{0}\right)\right) \exp \left(-m s_{1} \theta_{1} T+m s_{2} \theta_{2} T-m s_{3}\left(T-\theta_{1} T-\theta_{2} T\right)\right) .
$$

Because $\left(t-\theta_{1} T\right) / T<m \leq t / T$, then

$$
V(e(t)) \leq V\left(e\left(t_{0}\right)\right) \exp \left(-\left(s_{1} \theta_{1}-s_{2} \theta_{2}+s_{3}\left(1-\theta_{1}-\theta_{2}\right)\right)\left(t-\theta_{1} T\right)\right)
$$

Subcase 2. If $m T+\theta_{1} T \leq t<m T+\left(\theta_{1}+\theta_{2}\right) T$, then we have that

$$
V(e(t)) \leq V\left(e\left(t_{0}\right)\right) \exp \left(-(m+1) s_{1} \theta_{1} T+(m+1) s_{2} \theta_{2} T-m s_{3}\left(T-\theta_{1} T-\theta_{2} T\right)\right)
$$

Because $\left(t+T-\theta_{1} T-\theta_{2} T\right) / T<m+1 \leq\left(t+T-\theta_{1} T\right) / T$, then

$$
\begin{aligned}
V(e(t)) \leq & V\left(e\left(t_{0}\right)\right) \exp \left(-\left(s_{1} \theta_{1}-s_{2} \theta_{2}+s_{3}\left(1-\theta_{1}-\theta_{2}\right)\right)\left(t+T-\theta_{1} T-\theta_{2} T\right)\right. \\
& \left.+s_{3}\left(T-\theta_{1} T-\theta_{2} T\right)\right) .
\end{aligned}
$$


Subcase 3. If $m T+\left(\theta_{1}+\theta_{2}\right) T \leq t<(m+1) T$, similarly, then we have that

$$
V(e(t)) \leq V\left(e\left(t_{0}\right)\right) \exp \left(-\left(s_{1} \theta_{1}-s_{2} \theta_{2}+s_{3}\left(1-\theta_{1}-\theta_{2}\right)\right) t+s_{3}\left(T-\theta_{1} T-\theta_{2} T\right)\right)
$$

Therefore, in this situation, for any $t>0$, if $s_{1} \theta_{1}-s_{2} \theta_{2}+s_{3}\left(1-\theta_{1}-\theta_{2}\right)>0$, error system (11) is exponentially stable, which implies system (6) and system (8) with time delays can realize exponential synchronization.

Corollary 1 If there are positive scalars $\theta_{1}, \theta_{2}, s_{1}, s_{2}$ and $s_{3}$ that satisfy the condition

$$
s_{1}^{\prime} \theta_{1}-s_{2}^{\prime} \theta_{2}+s_{3}^{\prime}\left(1-\theta_{1}-\theta_{2}\right)>0
$$

where $0<\theta_{1}+\theta_{2}<1, s_{1} \leq s_{1}^{\prime}=-\lambda_{\min }\left(A^{T}+A\right)-\lambda_{\min }\left(B B^{T}\right)-2 I_{1}-\tilde{L}^{2}, s_{2} \geq s_{2}^{\prime}=\lambda_{\min }\left(A^{T}+A\right)+$ $\lambda_{\min }\left(B B^{T}\right)+\tilde{L}^{2}$, and $s_{3} \leq s_{3}^{\prime}=-\lambda_{\min }\left(A^{T}+A\right)-\lambda_{\min }\left(B B^{T}\right)-2 I_{2}-\tilde{L}^{2}$, then the memristor-based hyperchaotic systems (6) and (8) with time delays can realize exponential synchronization.

\section{Simulation results}

In this section, the simulation results will be displayed. Set $\gamma_{1}=15, \gamma_{2}=-3.2, \gamma_{3}=19.7$, $\gamma_{4}=1, \gamma_{5}=1, \gamma_{6}=15, \gamma_{7}=0.52$, and let these two systems get their initial values:

$$
\begin{aligned}
& \left(x_{1}(0), x_{2}(0), x_{3}(0), x_{4}(0)\right)^{T}=\left(10^{-6}, 10^{-6}, 0,0\right)^{T}, \\
& \left(y_{1}(0), y_{2}(0), y_{3}(0), y_{4}(0)\right)^{T}=\left(0,10^{-4}, 10^{-4}, 10^{-4}\right)^{T} .
\end{aligned}
$$

According to the boundaries of state variables, we get $\tilde{L}=4.1794$. When $I_{1}=-8, I_{2}=-7$, if we choose $T=1, \theta_{1}=0.3, \theta_{2}=0.2, s_{1}=5, s_{2}=4, s_{3}=5$ and $\tau=0.3$, then by Theorem 1 and Corollary 1 , we know that system (11) is exponentially stable. Synchronization between two memristor-based systems with $\tau=0.3$ is shown in Figure 3.



Figure 3 Sandwich synchronization between two memristor-based hyperchaotic systems with $\tau=0.3$. 


\section{Conclusions}

In this paper, the characteristics of a memristor-based hyperchaotic system have been discussed. Based on Lyapunov stability theory, matrix inequality, sandwich control and considering time delays, this paper designed one type of sandwich controller and applied it to realize the exponential synchronization between two memristor-based hyperchaotic systems with transmission time delays. Simulation results were given to verify the effectiveness of this method.

\section{Competing interests}

The authors declare that they have no competing interests.

\section{Acknowledgements}

The authors sincerely thank the referees for their helpful suggestions, which greatly improved the paper. This research is supported by Chongqing Municipal Key Laboratory of Institutions of Higher Education (Grant No. [2017]3) and Program of Chongqing Development and Reform Commission (Grant No. 2017[1007]).

\section{Competing interests}

The authors declare that they have no competing interests.

\section{Authors' contributions}

All authors read and approved the final manuscript.

\section{Author details}

${ }^{1}$ School of Computer Science and Engineering, Chongqing Three Gorges University, Wanzhou, Chongqing 404120,

P.R. China. ${ }^{2}$ School of Mathematics and Statistics, Chongqing Three Gorges University, Wanzhou, Chongqing 404120,

P.R. China.

\section{Publisher's Note}

Springer Nature remains neutral with regard to jurisdictional claims in published maps and institutional affiliations.

Received: 14 August 2017 Accepted: 14 December 2017 Published online: 16 January 2018

\section{References}

1. Chua, LO: Memristor - the missing circuit element. IEEE Trans. Circuit Theory 18(5), 507-519 (1971)

2. Strukov, DB, Snider, GS, Stewartand, DR, Williams, RS: The missing memristor found. Nature 453, 80-83 (2008)

3. Guckert, L, Swartzlander, EE: Optimized memristor-based multipliers. IEEE Trans. Circuits Syst. I, Regul. Pap. 64(2), 373-385 (2017)

4. Bao, B, Liu, Z, Xu, J: Transient chaos in smooth memristor oscillator. Chin. Phys. B 19(3), Article ID 030510 (2010)

5. Corinto, F, Ascoli, A, Gilli, M: Nonlinear dynamics of memristor oscillators. IEEE Trans. Circuits Syst. I, Regul. Pap. 58(6), 1323-1336 (2011)

6. Li, C, Ge, J, Tian, Y: Associative learning of memristive synapses circuits based on spiking neural networks. J. Chongqing Univ. 37(7), 115-124 (2014) (in Chinese)

7. Li, Z, Zeng, Y: A memristor oscillator based on a twin-T network. Chin. Phys. B 22(4), Article ID 040502 (2013)

8. Riaza, R: First order mem-circuits: modeling, nonlinear oscillations and bifurcations. IEEE Trans. Circuits Syst. I, Regul. Pap. 60(6), 1570-1583 (2013)

9. Talukdar, A, Radwan, AG, Salama, KN: Generalized model for memristor-based Wien family oscillators. Microelectron. J. 42(9), 1032-1038 (2011)

10. Talukdar, A, Radwan, AG, Salama, KN: Nonlinear dynamics of memristor based 3rd order oscillatory system. Microelectron. J. 43(3), 169-175 (2012)

11. Wu, A, Wen, S, Zeng, Z: Synchronization control of a class of memristor-based recurrent neural networks. Inf. Sci. 183(1), 106-116 (2012)

12. Wu, A, Zeng, Z: Dynamic behaviors of memristor-based recurrent neural networks with time-varying delays. Neural Netw. 36, 1-10 (2012)

13. Wu, A, Zeng, Z: Exponential stabilization of memristive neural networks with time delays. IEEE Trans. Neural Netw. Learn. Syst. 23(12), 1919-1929 (2012)

14. Itoh, M, Chua, LO: Memristor oscillators. Int. J. Bifurc. Chaos 18(11), 3183-3206 (2008)

15. Bao, B, Liu, Z, Xu, J: Steady periodic memristor oscillator with transient chaotic behaviours. Electron. Lett. 46(3), 237-238 (2010)

16. Bao, B, Xu, J, Liu, Z: Initial state dependent dynamical behaviors in memristor based chaotic circuit. Chin. Phys. Lett. 27(7), Article ID 070504 (2010)

17. Zhang, B, Deng, F, Zhao, X, Zhang, C: Hybrid control of stochastic chaotic system based on memristive Lorenz system with discrete and distributed time-varying delays. IET Control Theory Appl. 10(13), 1513-1523 (2016)

18. Min, G, Duan, S, Wang, L: A double-wing chaotic system based on ion migration memristor and its sliding mode control. Int. J. Bifurc. Chaos Appl. Sci. Eng. 26(8), Article ID 1650129 (2016) 
19. Ding, D, Qian, X, Hu, W, Wang, N, Liang, D: Chaos and Hopf bifurcation control in a fractional-order memristor-based chaotic system with time delay. Eur. Phys. J. Plus 132, Article ID 447 (2017). https://doi.org/10.1140/epjp/i2017-11699-9

20. Shen, W, Zeng, Z, Wang, G: Feedback stabilization of memristor-based hyper chaotic systems. In: Third International Conference on Information Science and Technology, 23-25 March, Yangzhou, Jiangsu, China (2013)

21. $\mathrm{Wu}, \mathrm{H}, \mathrm{Li}, \mathrm{R}$, Yao, R, Zhang, X: Weak, modified and function projective synchronization of chaotic memristive neural networks with time delays. Neurocomputing 149, 667-676 (2015)

22. $\mathrm{Wu}, \mathrm{H}, \mathrm{Li}, \mathrm{R}, \mathrm{Wei}, \mathrm{H}$, Zhang, X, Yao, R: Synchronization of a class of memristive neural networks with time delays via sampled-data control. Int. J. Mach. Learn. Cybern. 6(3), 365-373 (2015)

23. Huang, J, Li, C, He, X: Stabilization of a memristor-based chaotic system by intermittent control and fuzzy processing. Int. J. Control. Autom. Syst. 11(3), 643-647 (2013)

24. Feng, Y, Li, C, Huang, T: Sandwich control systems with impulse time windows. Int. J. Mach. Learn. Cybern. 8 2009-2015 (2017). https://doi.org/10.1007/s13042-016-0580-5

25. Feng, Y, Li, C, Huang, T: Sandwich control systems. In: Sixth International Conference on Intelligent Control and Information Processing (ICICIP), 26-28 November. IEEE, New York (2015). https://doi.org/10.1109/ICICIP.2015.7388134

26. Huang, T, Li, C, Liu, X: Synchronization of chaotic systems with delay using intermittent linear state feedback. Chaos 18, Article ID 033122 (2008)

27. Huang, T, Li, C, Duan, S, Starzyk, JA: Robust exponential stability of uncertain delayed neural networks with stochastic perturbation and impulse effects. IEEE Trans. Neural Netw. Learn. Syst. 23(6), 866-875 (2012)

28. Wen, S, Zeng, Z, Chen, MZQ, Huang, T: Synchronization of switched neural networks with communication delays via the event-triggered control. IEEE Trans. Neural Netw. Learn. Syst. 28(1), 2334-2343 (2017). https://doi.org/10.1109/TNNLS.2016.2580609

29. Tu, Z, Jian, J, Wang, K: Global exponential stability in Lagrange sense for recurrent neural networks with both time-varying delays and general activation functions via LMI approach. Nonlinear Anal., Real World Appl. 12(12), 2174-2182 (2011)

30. Bao, C, Liu, Z, Xu, J: Transient chaos in smooth memristor oscillator. Chin. Phys. B 19(3), Article ID 030510 (2010)

31. Wu, A: Hyperchaos synchronization of memristor oscillator system via combination scheme. Adv. Differ. Equ. 2014, Article ID 86 (2014). https://doi.org/10.1186/1687-1847-2014-86

32. Sanchez, EN, Perez, JP: Input-to-state stability (ISS) analysis for dynamic neural networks. IEEE Trans. Circuits Syst. I, Fundam. Theory Appl. 46(11), 1395-1398 (1999)

\section{Submit your manuscript to a SpringerOpen ${ }^{\circ}$ journal and benefit from:}

- Convenient online submission

- Rigorous peer review

- Open access: articles freely available online

- High visibility within the field

- Retaining the copyright to your article

Submit your next manuscript at $>$ springeropen.com 